\title{
ACÚMULO E DISTRIBUIÇÃO DE METAIS PESADOS NAS RAÍZES, CAULE E FOLHAS DE MUDAS DE ÁRVORES EM SOLO CONTAMINADO POR REJEITOS DE INDÚSTRIA DE ZINCO ${ }^{1}$
}

\section{CLÁUDIO ROBERTO FONSÊCA SOUSA SOARES ${ }^{2}$, ADRIANA MARIA DE AGUIAR ACCIOLY ${ }^{3}$, TERESA CRISTINA LARA LANZA DE SÁ E MELO MARQUES ${ }^{3}$, JOSÉ OSWALDO SIQUEIRA ${ }^{4}$, FÁTIMA MARIA SOUZA MOREIRA ${ }^{4}$.}

Departamento de Ciência do Solo, Universidade Federal de Lavras, Cx.P. 37, Lavras, MG, 37200-000.

RESUMO - Avaliaram-se em casa-de-vegetação o acúmulo e a distribuição de metais pesados nas raízes, caule e folhas de mudas de vinte espécies arbóreas tropicais crescendo em solo contaminado com metais pesados. As mudas com tamanho ideal para plantio foram transferidas para vasos contendo 3,3 $\mathrm{kg}$ de solo sem contaminação ou contaminado com $\mathrm{Zn}, \mathrm{Cd}, \mathrm{Cu}$ e $\mathrm{Pb}$, onde permaneceram por 90 dias. As espécies que cresceram em solo contaminado apresentaram teores foliares elevados, variando de $\left(\mathrm{mg} \mathrm{kg}^{-1}\right): \mathrm{Zn}=$ 154 a 1177; $\mathrm{Cd}=0,6$ a 54,6; e $\mathrm{Cu}=2,8$ a 134. Essas concentrações foram, em muitos casos, superiores aos níveis críticos de toxidez, ao passo que os teores foliares de $\mathrm{Pb}$ variaram de 0,1 a $4,3 \mathrm{mg} \mathrm{kg}{ }^{-1}$, não atingindo níveis considerados fitotóxicos. Em algumas espécies como Machaerium nictidans, Myroxylon peruiferum, Piptadenia gonoacantha, Senna macranthera e Trema micrantha, que tiveram o crescimento muito inibido pela contaminação, houve elevada translocação de $\mathrm{Zn}$ e/ou Cd para parte aérea. Dendropanax cuneatum, que foi pouco afetada pelos metais, também apresentou índice elevado de translocação de $\mathrm{Zn}$ e Cd, mas reteve esses elementos no caule. Acacia mangium, Copaifera langsdorffi e Cedrella fissilis também apresentaram baixa sensibilidade à contaminação e apresentaram elevado acúmulo de $\mathrm{Zn}$ e $\mathrm{Cd}$ nas raízes, indicando que a baixa translocação desses elementos para a parte aérea está envolvida na tolerância dessas espécies ao excesso de metais pesados no solo. $O$ padrão de distribuição proporcional de $\mathrm{Zn}$ e $\mathrm{Cd}$ nas raízes e parte aérea das plantas se relaciona com o comportamento dessas ao excesso de metais pesados no solo.

TERMOS ADICIONAIS PARA INDEXAÇÃO: Toxicidade de metais pesados, níveis críticos, poluição do solo, mudas de árvores tropicais.

\footnotetext{
${ }^{1}$ Recebido: 28/2/2001 - Aceito: 10/10/2001 - Trabalho financiado pelo convênio CMM/FAEPE e FAPEMIG

${ }^{2}$ Eng. Agrôn., MS, crfsousa@ufla.br. Bolsista FAPEMIG

${ }^{3}$ Eng. Agrôn., MS, Dept. de Ciência do Solo, Universidade Federal de LavraS (UFLA), Caixa Postal 37, 37200-000, Lavras MG.

${ }^{4}$ Prof. titular, PhD, Dept. de Ciência do Solo, Universidade Federal de Lavras (UFLA), Caixa Postal 37, 37200-000, Lavras MG. Bolsista do CNPq
} 


\title{
CONTENT AND DISTRIBUTION OF HEAVY METALS IN ROOTS, STEMS AND LEAVES OF TREE SEEDLINGS IN SOIL CONTAMINATED BY ZINC INDUSTRY WASTES
}

\begin{abstract}
An experiment was carried out in greenhouse to evaluate total content and distribution of heavy metals in seedlings of twenty different tree species growing in a soil contaminated by $\mathrm{Zn}, \mathrm{Cd}, \mathrm{Cu}$ and $\mathrm{Pb}$. Plantable size seedlings were transfered to $3.3 \mathrm{~kg}$ pots containing a contaminated soil mixture and to a control soil without contamination where they were allowed to grow for ninety days. Metal foliar concentrations for plants grown in the contaminated soil were high, ranged from $\left(\mathrm{mg} \mathrm{kg}^{-1}\right): \mathrm{Zn}=154$ to $1177 ; \mathrm{Cd}=0.6$ to 54.6 and $\mathrm{Cu}=2.8$ to 134 . In most cases, these concentrations were superior to what has been considered as critical toxic levels, whereas foliar $\mathrm{Pb}$ concentrations ranged from 0.1 to $4.3 \mathrm{mg} \mathrm{kg}^{-1}$, below the critical toxic level. In some species that were highly affected by contamination such as Machaerium nictidans, Myroxylon peruiferum, Piptadenia gonoacantha, Senna macranthera and Trema micrantha it was found high translocation index for $\mathrm{Zn}$ and/or Cd. However, Dendropanax cuneatum that was only slightly affected by contamination, exhibited high translocation of $\mathrm{Zn}$ and $\mathrm{Cd}$ to shoots. In contrast to others species, Dendropanax cuneatum retained these elements in the stems. Other group of plants that were only slightly affected by soil contamination such as Acacia mangium, Copaifera langsdorffi and Cedrella fissilis accumulated more $\mathrm{Zn}$ and $\mathrm{Cd}$ in the roots than in the shoots, therefore indicating that reduced translocation is involved in their tolerance to the excess of heavy metal in soil. The proportional distribution pattern of $\mathrm{Zn}$ and $\mathrm{Cd}$ in the roots and shoots of the studied plant species is related to their behavior to the excess of heavy metals in soil.
\end{abstract}

INDEX TERMS: Heavy metal toxicity, critical levels, soil pollution, tropical tree seedlings.

\section{INTRODUÇÃO}

Apesar do grande interesse pelos problemas ecológicos e ambientais causados pelo excesso de metais pesados no sistema solo-planta, pouca ênfase tem sido dada ao impacto desses elementos sobre a flora arbórea tropical. Apesar das espécies lenhosas apresentarem menor grau de tolerância a níveis tóxicos de metais pesados do que espécies herbáceas (Eltrop et al., 1991), o uso das primeiras na recuperação de áreas contaminadas é interessante, considerando que os metais absorvidos ficarão por mais tempo imobilizados nos tecidos vegetais, principalmente no caule, retardando o retorno desses elementos ao solo. Para isso, é necessário conhecer a capacidade de absorção, acúmulo e distribuição de metais pesados em espécies arbóreas, pois estudos dessa natureza são de grande importância para se obter sucesso em programas de fitorremediação (Huang e Cunningham, 1996). Além dessas características, a recuperação de solos contaminados por metais pesados requer a adoção de técnicas para amenizar a biodisponibilidade dos metais (Accioly e Siqueira, 2000) e o emprego de ecótipos tolerantes à contaminação capazes de sobreviver e completar o seu ciclo de vida (Shaw, 1989).

Com exceção das espécies exclusoras, a maioria das espécies vegetais crescendo em solos contaminados por metais pesados não consegue evitar a absorção desses elementos, mas somente limitar sua translocação (Baker, 1981). Ainda que existam muitas incertezas sobre a especificidade dos mecanismos de absorção dos metais pesados, sobretudo daqueles não essenciais, geralmente o teor e o acúmulo do elemento nos tecidos são funções de sua disponibilidade na solução do solo, e os teores nas raízes e parte aérea aumentam com a elevação da concentração de metais na solução do solo (Gussarsson et al., 1995). Os metais absorvidos sofrem um transporte radial na raiz, fundamentalmente apoplástico, encontrando um primeiro filtro de difusão e de regulação na endoderme. Já no estelo, os metais seguem 
essencialmente via xilema e, em suas relações com as células vizinhas, podem induzir alterações na diferenciação do próprio sistema vascular, uma vez que, em concentrações menores, alcançam as folhas, podendo alterar a estrutura e a funcionalidade das células fotossintéticas (Barceló e Poschenrieder, 1992). Espécies ou variedades de uma mesma espécie vegetal, expostas a uma concentração similar de metais pesados, podem diferir na absorção e/ou distribuição interna desses na planta. Isso pode resultar em diferenças na capacidade de retenção do elemento absorvido nas raízes, e/ou variação na carga no xilema (Shaw, 1989). Outros fatores, tais como estágio de desenvolvimento da planta, tempo de exposição ao metal $\mathrm{e}$ as diferentes espécies químicas dos elementos, podem também interferir nesses aspectos, refletindo nos teores dos metais nas diferentes partes da planta (Alloway, 1993). A regulação da absorção de metais pesados da rizosfera, o acúmulo desses nas raízes, preservando sua integridade e funções primárias, e a baixa translocação para a parte aérea são considerados mecanismos pelos quais o sistema radicular pode contribuir para a tolerância de espécies arbóreas a metais pesados (Verkleij e Parest, 1989; Arduini et al., 1996).

Neste trabalho, avaliaram-se os teores, acúmulo e distribuição de metais pesados nas raízes, caule e folhas de mudas de vinte espécies arbóreas transplantadas para mistura de solo contaminado por diversos metais pesados.

\section{MATERIAL E MÉTODOS}

O experimento foi desenvolvido em casade-vegetação no Departamento de Ciência do Solo da Universidade Federal de Lavras (UFLA), com mudas formadas em viveiro e transplantadas para vasos no período de janeiro a abril de 1996. Amostras de um solo contaminado por metais pesados foram coletadas em área de rejeito da Companhia Mineira de Metais (CMM) no município de Três Marias (MG), cuja contaminação ocorre pela deposição de rejeitos de indústria de processamento de zinco, que são misturados ao solo por vários anos (Ribeiro-Filho, 1999). O tratamento-controle consistiu de um Latossolo Vermelho-Escuro (LE) não contaminado, coletado na camada superficial (0-20 $\mathrm{cm})$ no município de São Sebastião da Vitória (MG). Devido ao elevado grau de contaminação do solo contaminado (teores totais em mg kg-1 de solo: $\mathrm{Zn}=18600 ; \mathrm{Cd}=135 ; \mathrm{Pb}=600$ e $\mathrm{Cu}=596$, além de outros em menores teores), este foi misturado na proporção de $40 \%(\mathrm{v} / \mathrm{v})$ com o LE não contaminado $(60 \% \mathrm{v} / \mathrm{v})$, mistura essa capaz de permitir o crescimento de mudas de espécies arbóreas nativas (Marques et al., 2000). Os solos foram secos ao ar e passados em peneira de malha $5 \mathrm{~mm}$, e com base na análise química, procedeu-se à correção de acidez com $\mathrm{Ca}(\mathrm{OH})_{2}$ puro e do nível de $\mathrm{P}$ do solo com superfosfato triplo (200 $\left.\mathrm{mg} \mathrm{kg}^{-1}\right)$, de forma a elevar a saturação por base e nível de $\mathrm{P}$ do solo não contaminado (LE) a valores próximos ao solo contaminado. O LE foi incubado por 15 dias, com a umidade em torno de $70 \%$ do volume total de poros (VTP), e após esse período, ambos os solos receberam vermicomposto $(10 \%$ por peso úmido), transferindo os mesmos para vasos plásticos com capacidade de $3,3 \mathrm{~kg}$ de solo. Amostras foram retiradas de ambos os solos para análises químicas, conforme Embrapa (1979), dos teores totais de metais pesados após digestão com água régia, conforme Foster (1995), e determinação por espectrofotometria de absorção atômica, cujos resultados encontram-se na Tabela 1.

O delineamento experimental foi inteiramente casualizado, com três repetições, em esquema fatorial $20 \times 2$, sendo 20 espécies vegetais e duas situações de solo. As espécies utilizadas são, em sua grande maioria, nativas do Sudeste brasileiro e foram selecionadas considerando-se a disponibilidade de mudas e o potencial da espécie em programas de recomposição florística de áreas degradadas (Botelho et al., 1995). Empregaram-se mudas formadas em viveiro, em sacos plásticos, com substrato composto de terra de subsolo misturado com palha de arroz carbonizada, sendo transplantada uma muda por vaso. A idade das 
TABELA 1. Características químicas do solo contaminado e não contaminado (controle) utilizados para o crescimento das mudas de espécies arbóreas.

\begin{tabular}{|c|c|c|c|c|c|c|c|c|c|c|c|c|c|}
\hline SOLO & $\begin{array}{c}\mathrm{pH} \\
\left(\mathrm{H}_{2} \mathrm{O}\right)\end{array}$ & $\begin{array}{l}\text { Mat. } \\
\text { Org. } \\
\mathrm{g} \mathrm{kg}^{-1}\end{array}$ & $\begin{array}{c}\text { (P } \\
\mathrm{mg}\end{array}$ & $\begin{array}{l}\mathrm{K})^{\mathrm{a}} \\
\mathrm{kg}^{-1}\end{array}$ & $\mathrm{Ca}$ & $\mathrm{Mg}$ & $1_{\mathrm{c}} \mathrm{dm}^{-3}$ & $\mathrm{Al}$ & $(\mathrm{Cd}$ & $\mathrm{Cu}$ & $\begin{array}{c}\mathrm{Zn} \\
\mathrm{mg} \mathrm{kg}{ }^{-1}\end{array}$ & $\mathrm{Mn}$ & $\mathrm{Pb})^{\mathrm{b}}$ \\
\hline Controle & 6,6 & 43 & 27 & 120 & 31 & 17 & 26 & 1 & 2 & 30 & 76 & 100 & 20 \\
\hline Contaminado & 6,3 & 35 & 43 & 103 & 26 & 19 & 21 & 1 & 75 & 1.000 & 8.800 & 586 & 427 \\
\hline
\end{tabular}

a/ Extraído por Mehlich

b/ Digestão com água régia conforme Foster (1995)

mudas variou de 6 a 13 meses em função do desenvolvimento de cada espécie no viveiro, atingindo-se o tamanho adequado para plantio das mesmas. As espécies estudadas e suas respectivas famílias encontram-se a seguir: Acacia mangium L. (Mimosaceae), Peltophorum dubium (Spreng) Taub. (Caesalpiniaceae), Anadenanthera peregrina (Benth.) Speg. (Mimosaceae), Machaerium nictidans Benth. (Fabaceae), Cedrella fissilis Vell (Meliaceae), Casearia lasiophylla Eichler (Flacourtiaceae), Senna macranthera (Vell.) I e B. (Caesalpiniaceae), Solanum granulosum-leprosum Dun. (Solanaceae), Tabebuia impetiginosa (Mart.) Standl. (Bignoniaceae), Platypodium gonoacantha (Mart.) Macbr. (Fabaceae), Piptadenia gonoacantha (Mart.) Macbr. (Mimosaceae), Hymenaea courbaril L. (Caesalpiniaceae), Cordia trichotoma (Vell.) Arrab. (Boraginaceae), Dendropanax cuneatum (DC.) Dene e Planch (Araliaceae), Myroxylon peruiferum L. (Fabaceae), Copaifera langsdorffii Desf. (Caesalpiniaceae), Myrsine umbellata Mart. (Myrsinaceae), Mimosa caesalpiniaefolia Benth. (Mimosaceae), Sebastiana schottiana (Euphorbiaceae) e Trema micrantha Blume (Ulmaceae). No transplante, o substrato aderido à muda foi eliminado por meio de lavagens para reduzir a interferência na resposta das plantas à contaminação. $\mathrm{O}$ experimento foi conduzido por 90 dias, em casa-de-vegetação, mantendo-se o solo com umidade próxima de $60 \%$ da VTP. Após esse período, as plantas foram colhidas e separadas em raízes, caule e folhas, secas em estufa com circulação de ar a $70^{\circ} \mathrm{C}$, pesadas e moídas. Posteriormente, efetuou-se a digestão nítrico-perclórica do material para determinação nos extratos de $\mathrm{Zn}, \mathrm{Cd}, \mathrm{Cu}$ e $\mathrm{Pb}$ por espectrofotometria de absorção atômica. Em vista dos elevados teores de $\mathrm{Zn}$ na matéria seca e da necessidade de diluições, optou-se pelas leituras dos teores desse elemento por espectrometria de emissão atômica, com plasma induzido em argônio (ICP/AES). Com base nas concentrações e produção de matéria seca, calcularam-se as quantidades acumuladas dos metais pesados nas raízes, caule e folhas. Por meio da quantidade acumulada, calculou-se a porcentagem de acúmulo de metais pesados para cada parte da planta em relação ao acúmulo total e o índice de translocação dos elementos, de acordo com Abichequer e Bohnen (1998), pela seguinte equação:

IT $=\frac{\text { Quantidade acumulada na parte aérea (folhas }+ \text { caule) }}{\text { Quantidade acumulada na planta }}$
Os dados foram submetidos à análise de variância e testes de médias pelo programa estatístico SAS (SAS Institute Inc.). 


\section{RESULTADOS E DISCUSSÃO}

A produção de matéria seca de raízes, caule e folhas das espécies arbóreas foi influenciada de modo diferenciado pela contaminação do solo (Figura 1). Verifica-se que apenas $A$. mangium, C. langsdorffii, D. cuneatum e T. impetiginosa foram as espécies que não apresentaram redução na matéria seca de raízes, caule e folhas, indicando a menor sensibilidade dessas espécies à contaminação; ao passo que $A$. peregrina, C. lasiophylla, M. caesalpiniaefolia e $S$. schotianna mostraram-se altamente sensíveis à contaminação, apresentando decréscimo significativo em todas as partes da planta. Para as demais espécies, verifica-se que a contaminação do solo reduziu significativamente a produção de matéria seca das folhas, com exceção de $C$. fissilis, $M$. nictidans e $S$. granulosum-leprosum, que apresentaram redução apenas nas raízes e/ou caule. Portanto, o comportamento das espécies foi bastante variável, o que torna extremamente complexa e difícil a determinação de um valor médio que reflita o grau de contaminação do solo em um ecossistema de mata nativa.

Os teores de $\mathrm{Zn}, \mathrm{Cd}, \mathrm{Cu}$ e $\mathrm{Pb}$ nas raízes, caule e folhas das plantas foram significativamente influenciados pela contaminação do solo, sendo esse efeito diferenciado para espécie e metais (Tabelas 2 e 3). De maneira geral, houve aumento nos teores de $\mathrm{Zn}$ em todas as partes das plantas que se desenvolviam em solo contaminado (Tabela 2), verificando-se incrementos de até $10.735 \%$ nas raízes de $H$. courbaril, $6.043 \%$ no caule de $S$. granulosum-leprosum e $1.946 \%$ nas folhas de $C$. trichotoma, em relação ao controle. Em virtude da elevada concentração de $\mathrm{Zn}$ no solo (Tabela 1), verifica-se que 12 das 20 espécies estudadas apresentaram teor na matéria seca das folhas maior que o limite superior da faixa tóxica de 100 a 400 mg kg${ }^{-1}$ adotada por Kabata-Pendias e Pendias (1985), indicando o alto potencial fitotóxico desse elemento para as plantas. $C$. trichotoma e $S$. macranthera foram as espécies que apresentaram os maiores teores de $\mathrm{Zn}$ nas folhas. $\mathrm{O}$ teor de $\mathrm{Cd}$ foi muito baixo em todas as partes da planta desenvolvida no solo-controle (Tabela 2), ao passo em solo contaminado verificaram-se teores máximos, em mg kg${ }^{-1}$, de 213 (raízes), 58 (caule) e 55 (folhas) em $A$. mangium, $D$. cuneatum e $S$. granulosum-leprosum, respectivamente. A maioria das espécies apresentou teor de $\mathrm{Cd}$ nas folhas entre 5 e $30 \mathrm{mg} \mathrm{kg}^{-1}$, valores considerados fitotóxicos (Kabata-Pendias e Pendias, 1985), indicando que esse elemento também foi responsável pela redução do crescimento das espécies estudadas. Contudo, mesmo espécies que apresentaram baixos teores de $\mathrm{Cd}$ nas folhas, como $H$. courbaril e $M$. umbellata, apresentaram redução no crescimento em solo contaminado (Figura 1), mostrando que a multicontaminação e as possíveis interações químicas no solo e fisiológicas na planta devem ser consideradas para avaliar o impacto do excesso de metais pesados sobre as espécies (Barceló e Poschenrieder, 1992). Como as espécies cresceram no solo contendo níveis elevados de outros metais, principalmente $\mathrm{Zn}$, pode ter ocorrido efeito fitotóxico aditivo, como discutido em Kahle (1993).

A contaminação do solo também influenciou os teores de $\mathrm{Cu}$ e $\mathrm{Pb}$ na matéria seca de raízes, caule e folhas das espécies estudadas (Tabela 3). A maioria das espécies apresentou elevação nos teores de $\mathrm{Cu}$ na matéria seca das raízes desenvolvidas em solo contaminado, com valores variando entre $7,6 \mathrm{mg} \mathrm{kg}^{-1}(H$. courbaril) e $358 \mathrm{mg} \mathrm{kg}^{-1}$ (Piptadenia gonoacantha), os quais foram superiores aos teores verificados na parte aérea das plantas. Ao contrário de $\mathrm{Zn}, \mathrm{Cd}$ e $\mathrm{Cu}$, os teores de $\mathrm{Pb}$ nos tecidos foram pouco influenciados pela contaminação e apresentaram valores muito baixos. Apenas $C$. trichotoma e $D$. cuneatum apresentaram teores superiores a $2,0 \mathrm{mg} \mathrm{kg}^{-1}$ na matéria seca das folhas (Tabela 3), os quais são muito inferiores à faixa de $30-300 \mathrm{mg} \mathrm{kg}^{-1}$, considerada fitotóxica (Kabata-Pendias e Pendias, 1985) indicando, portanto, que $\mathrm{Pb}$ não é problema nesse solo.

A contaminação do solo exerceu grande influência sobre o acúmulo de $\mathrm{Zn}$ nos tecidos das espécies (Figura 2), principalmente por causa do incremento dos teores de $\mathrm{Zn}$ observados nos tecidos das plantas (Tabela 2). O acúmulo total de 

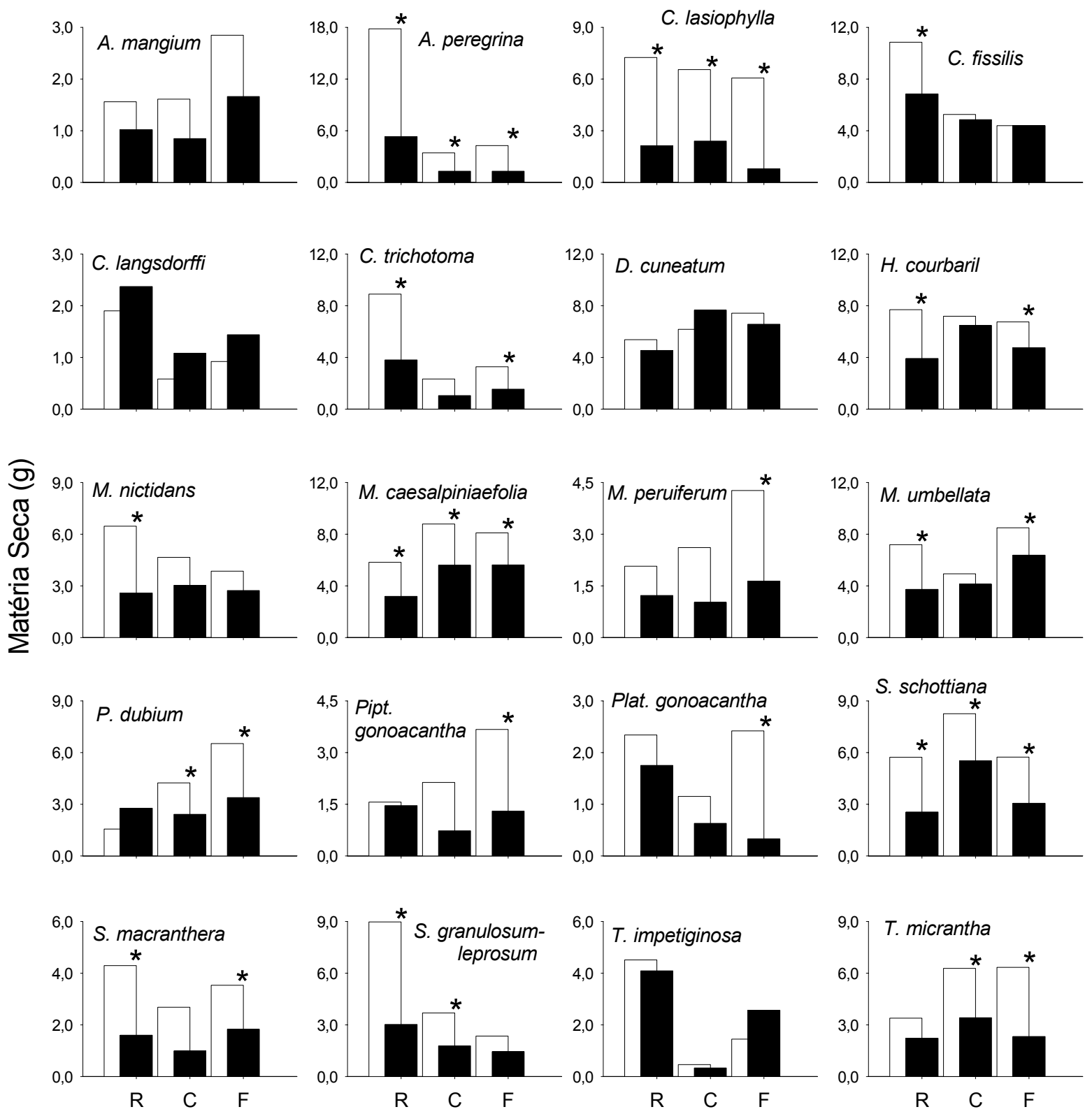

Controle

Solo Contaminado

FIGURA 1. Matéria seca das raízes $(\mathrm{R})$, caule $(\mathrm{C})$ e folhas $(\mathrm{F})$ de mudas de espécies arbóreas em solo contaminado e não contaminado (controle). * Efeito significativo da contaminação $(\mathrm{P} \leq 5 \%)$ 
Soares et al.

TABELA 2 - Teores de Zinco e Cádmio $\left(\mathrm{mg} \mathrm{kg}^{-1}\right)$ na raiz, caule e folhas de mudas de espécies arbóreas em solo com $(+)$ e sem (-) contaminação com metais pesados

\begin{tabular}{|c|c|c|c|c|c|c|c|}
\hline \multirow{2}{*}{ Espécies } & \multirow{2}{*}{ Contam. } & \multicolumn{3}{|c|}{ Zinco } & \multicolumn{3}{|c|}{ Cádmio } \\
\hline & & Raiz & Caule & Folhas & Raiz & Caule & Folhas \\
\hline \multirow{2}{*}{ A. mangium } & - & 98 & 58 & 65 & 0,2 & 0,0 & 0,1 \\
\hline & + & $3300 *$ & $691 *$ & $614 *$ & $213 *$ & $8,7 *$ & $7,2 *$ \\
\hline \multirow{2}{*}{ A. peregrina } & - & 108 & 31 & 35 & 0,5 & 0,6 & 0,2 \\
\hline & + & 384 & $273 *$ & $375 *$ & 16 & $6,1 *$ & $7,1 *$ \\
\hline \multirow{2}{*}{ C. lasiophylla } & - & 90 & 55 & 56 & 0,3 & 0,1 & 0,3 \\
\hline & + & $1356 *$ & $648 *$ & $461 *$ & $58 *$ & $15 *$ & $6,2 *$ \\
\hline \multirow[b]{2}{*}{ C. fissilis } & - & 109 & 60 & 88 & 0,1 & 0,2 & 0,2 \\
\hline & + & $870 *$ & 171 & $341 *$ & $54 *$ & $6,1 *$ & $6,3 *$ \\
\hline \multirow{2}{*}{ C. langsdorffi } & - & 84 & 82 & 83 & 0,5 & 0,8 & 0,3 \\
\hline & + & $819 *$ & $299 *$ & $324 *$ & $42 *$ & $7,3 *$ & $10 *$ \\
\hline \multirow{2}{*}{ C. trichotoma } & - & 48 & 123 & 57 & 0,1 & 1,7 & 0,2 \\
\hline & + & $2055 *$ & $1040 *$ & $1109 *$ & $55 *$ & $37 *$ & $16 *$ \\
\hline \multirow{2}{*}{ D. cuneatum } & - & 91 & 425 & 53 & 1,1 & 1,7 & 0,1 \\
\hline & + & $1569 *$ & $674 *$ & $443 *$ & 9,3 & $58 *$ & $33 *$ \\
\hline \multirow{2}{*}{ H. courbaril } & - & 17 & 70 & 46 & 0,0 & 0,9 & 0,1 \\
\hline & + & $1825 *$ & $256 *$ & $341 *$ & $39 *$ & $7,5 *$ & 2,4 \\
\hline \multirow{2}{*}{ M. nictidans } & - & 78 & 77 & 68 & 0,3 & 0,2 & 0,2 \\
\hline & + & $1808 *$ & $1092 *$ & $725 *$ & $45 *$ & $33 *$ & $37 *$ \\
\hline \multirow{2}{*}{ M. caesalpiniaefolia } & - & 262 & 105 & 90 & 0,2 & 0,0 & 0,0 \\
\hline & + & $1842 *$ & 171 & $350 *$ & $81 *$ & $8,3 *$ & $7,1 *$ \\
\hline \multirow{2}{*}{ M. peruiferum } & - & 36 & 38 & 45 & 0,3 & 0,2 & 0,1 \\
\hline & + & $623^{*}$ & $614 *$ & $537 *$ & 17 & 2,0 & $7,8 *$ \\
\hline \multirow{2}{*}{ M. umbellata } & - & 66 & 202 & 242 & 0,2 & 0,0 & 0,1 \\
\hline & + & $1245 *$ & $384 *$ & 171 & $30 *$ & $6,7 *$ & 0,6 \\
\hline \multirow{2}{*}{ P. dubium } & - & 72 & 48 & 51 & 0,5 & 0,1 & 0,0 \\
\hline & + & $3104 *$ & $239 *$ & $878 *$ & $126 *$ & $12 *$ & $26 *$ \\
\hline \multirow{2}{*}{ Pipt. gonoacantha } & - & 54 & 88 & 52 & 0,8 & 0,5 & 0,0 \\
\hline & + & 333 & $333 *$ & $768 *$ & $157 *$ & $10 *$ & $22 *$ \\
\hline \multirow{2}{*}{ Plat. gonoacantha } & - & 70 & 78 & 100 & 1,9 & 1,1 & 0,3 \\
\hline & + & 375 & $384 *$ & 154 & 11 & $14 *$ & $7,6 *$ \\
\hline \multirow{2}{*}{ S. schottiana } & - & 103 & 78 & 110 & 0,6 & 0,2 & 0,2 \\
\hline & + & $1680 *$ & 171 & 230 & $100 *$ & $10 *$ & 4,8 \\
\hline \multirow{2}{*}{ S. macranthera } & - & 84 & 96 & 110 & 0,5 & 0,5 & 0,8 \\
\hline & + & $2805 *$ & $1211 *$ & $1177 *$ & $25 *$ & $31 *$ & $35 *$ \\
\hline \multirow{2}{*}{ S. granulosum-leprosum } & - & 58 & 35 & 94 & 0,5 & 0,1 & 4,4 \\
\hline & + & $3087 *$ & $2115^{*}$ & $802 *$ & $128 *$ & $43 *$ & $55 *$ \\
\hline \multirow{2}{*}{ T. impetiginosa } & - & 98 & 133 & 344 & 1,7 & 0,8 & 0,4 \\
\hline & + & $529 *$ & $435 *$ & 503 & 17 & $12 *$ & $6,9 *$ \\
\hline \multirow{2}{*}{ T. micrantha } & - & 112 & 35 & 50 & 0,4 & 0,1 & 0,1 \\
\hline & + & $1671 *$ & $631 *$ & $836 *$ & $63 *$ & $16 *$ & $22 *$ \\
\hline
\end{tabular}

* Efeito significativo da contaminação $(\mathrm{P} \leq 5 \%)$ 
TABELA 3 - Teores de Cobre e Chumbo $\left(\mathrm{mg} \mathrm{kg}^{-1}\right)$ na raiz, caule e folhas de mudas de espécies arbóreas em solo com (+) e sem (-) contaminação com metais pesados.

\begin{tabular}{|c|c|c|c|c|c|c|c|}
\hline \multirow{2}{*}{ Espécies } & \multirow{2}{*}{ Contam. } & \multicolumn{3}{|c|}{ Cobre } & \multicolumn{3}{|c|}{ Chumbo } \\
\hline & & Raiz & Caule & Folhas & Raiz & Caule & Folhas \\
\hline \multirow{2}{*}{ A. mangium } & - & 50 & 36 & 40 & 0,1 & 0,1 & 0,1 \\
\hline & + & $215 *$ & $7,7 *$ & 26 & 5,6 & 0,1 & 0,5 \\
\hline \multirow{2}{*}{ A. peregrina } & - & 22 & 16 & 14 & 0,1 & 1,0 & 0,1 \\
\hline & + & $69 *$ & 26 & 34 & 0,1 & 0,1 & 0,1 \\
\hline \multirow{2}{*}{ C. lasiophylla } & - & 63 & 41 & 41 & 1,4 & 11 & 0,5 \\
\hline & + & $120 *$ & 50 & $98 *$ & 2,2 & $1,3 *$ & 1,3 \\
\hline \multirow{2}{*}{ C. fissilis } & - & 66 & 41 & 63 & 0,1 & 0,1 & 1,4 \\
\hline & + & 74 & 46 & 49 & 0,1 & 0,1 & 0,1 \\
\hline \multirow{2}{*}{ C. langsdorffi } & - & 59 & 48 & 50 & 0,1 & 0,1 & 0,1 \\
\hline & + & $140 *$ & 46 & 49 & 3,9 & 0,5 & 0,1 \\
\hline \multirow{2}{*}{ C. trichotoma } & - & 22 & 40 & 27 & 0,1 & 0,1 & 0,1 \\
\hline & + & $122 *$ & 45 & $89 *$ & 5,6 & 0,1 & $4,3 *$ \\
\hline \multirow{2}{*}{ D. cuneatum } & - & 63 & 398 & 40 & 1,0 & 0,1 & 1,0 \\
\hline & + & $129 *$ & $64 *$ & 55 & 3,9 & 1,8 & 2,2 \\
\hline \multirow{2}{*}{ H. courbaril } & - & 16 & 19 & 7,2 & 0,1 & 0,1 & 0,1 \\
\hline & + & $358 *$ & 11 & 11 & 8,2 * & 0,9 & 0,1 \\
\hline \multirow{2}{*}{ M. nictidans } & - & 41 & 37 & 33 & 0,5 & 0,1 & 0,1 \\
\hline & + & $114 *$ & 36 & 36 & 1,7 & 0,1 & 1,3 \\
\hline \multirow{2}{*}{ M. caesalpiniaefolia } & - & 41 & 35 & 41 & 0,1 & 0,1 & 0,1 \\
\hline & + & $158 *$ & 35 & 31 & 3,0 & 0,1 & 0,1 \\
\hline \multirow{2}{*}{ M. peruiferum } & - & 9,0 & 5,0 & 5,0 & 0,1 & 0,1 & 0,1 \\
\hline & + & $43 *$ & 6,0 & 6,0 & 0,9 & 0,1 & 0,5 \\
\hline \multirow{2}{*}{ M. umbellata } & - & 28 & 42 & 35 & 0,1 & 0,1 & 0,1 \\
\hline & + & $97 *$ & $11 *$ & $6,0 *$ & 2,2 & 0,1 & 0,1 \\
\hline \multirow{2}{*}{ P. dubium } & - & 34 & 22 & 20 & 0,1 & 0,1 & 0,1 \\
\hline & + & $350 *$ & $102 *$ & $80 *$ & $9,1 *$ & 0,1 & 0,5 \\
\hline \multirow{2}{*}{ Pipt. gonoacantha } & - & 24 & 40 & 35 & 0,5 & 0,1 & 0,1 \\
\hline & + & 7,6 & $4,6 *$ & $5,7 *$ & 2,6 & $7,8 *$ & 1,7 \\
\hline \multirow{2}{*}{ Plat. gonoacantha } & - & 54 & 56 & 103 & 1,0 & 1,4 & 0,5 \\
\hline & + & 72 & 45 & $134 *$ & 0,9 & 1,3 & 0,1 \\
\hline \multirow{2}{*}{ S. schottiana } & - & 53 & 46 & 48 & 0,1 & 0,1 & 0,1 \\
\hline & + & $180 *$ & 39 & 37 & 4,3 & 0,1 & 0,1 \\
\hline \multirow{2}{*}{ S. macranthera } & - & 115 & 121 & 118 & 0,1 & 0,1 & 0,1 \\
\hline & + & $255 *$ & $7,4 *$ & $2,8 *$ & 6,1 & 0,1 & 0,1 \\
\hline \multirow{2}{*}{ S. granulosum-leprosum } & - & 31 & 7,8 & 29 & 0,1 & 0,1 & 0,1 \\
\hline & + & $147 *$ & 15 & 30 & 3,5 & $2,2 *$ & 1,3 \\
\hline \multirow{2}{*}{ T. impetiginosa } & - & 44 & 54 & 55 & 1,4 & 0,1 & 3,7 \\
\hline & + & 77 & 42 & 39 & $38 *$ & $17 *$ & $0,9 *$ \\
\hline \multirow{2}{*}{ T. micrantha } & - & 44 & 33 & 41 & 0,1 & 0,1 & 0,5 \\
\hline & + & $160 *$ & 27 & 30 & 5,2 & 0,9 & 0,9 \\
\hline
\end{tabular}

* Efeito significativo da contaminação $(\mathrm{P} \leq 5 \%)$ 


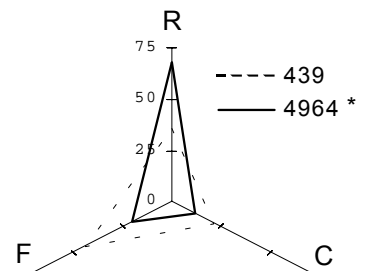

A. mangium

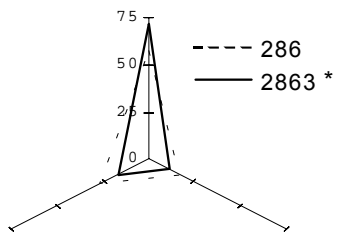

C. langsdorffi

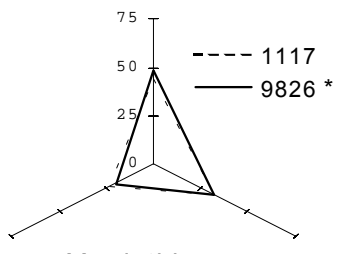

M. nictidans

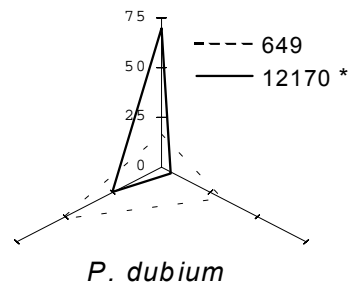

P. dubium

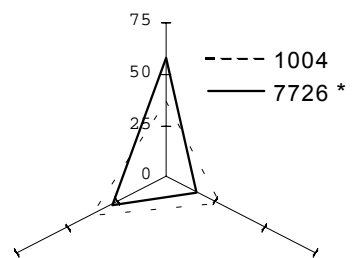

S. macranthera

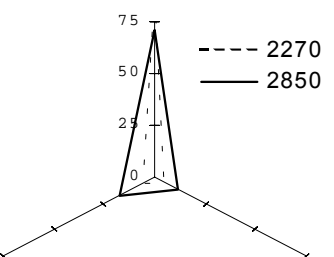

A. peregrina

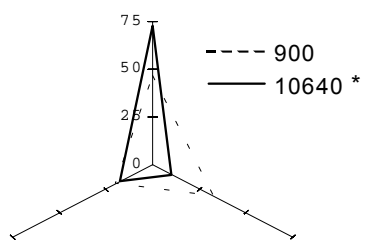

C. trichotoma

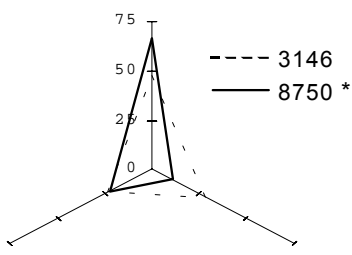

M. caesalpiniaefolia

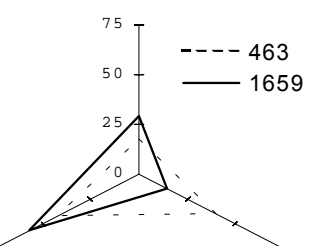

Pipt. gonoacantha

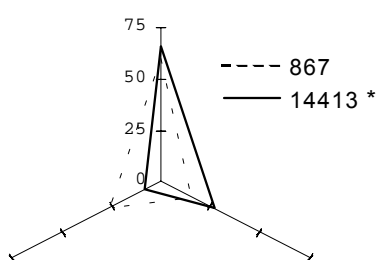

S. granulosum-leprosum

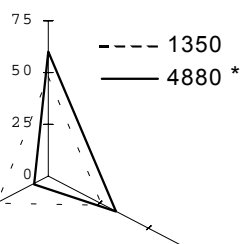

C. lasiophylla

C. fissilis

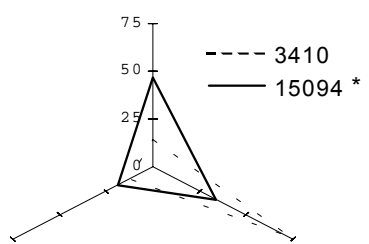

D. cuneatum

H. courbaril

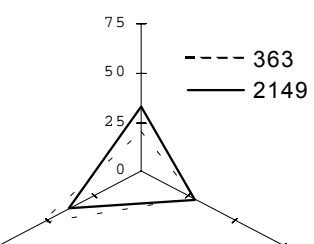

M. peruiferum
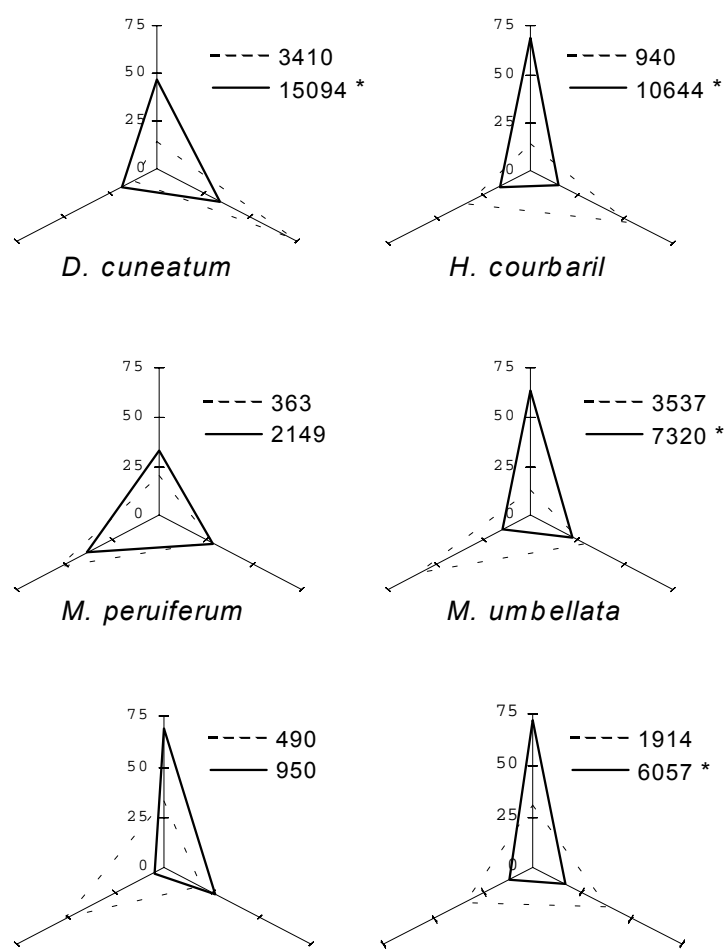

Plat. gonoacantha

S. schottiana

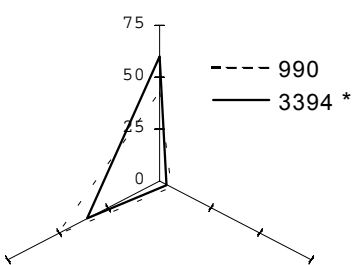

T. impetiginosa

T. micrantha

FIGURA 2. Acúmulo e distribuição (\% do total acumulado) de $\mathrm{Zn}$ nas raízes $(\mathrm{R})$, caule $(\mathrm{C})$ e folhas $(\mathrm{F}) \mathrm{em}$ mudas de espécies arbóreas. Linhas tracejadas e contínuas representam o total acumulado $\left(\mu \mathrm{g}\right.$ planta $\left.^{-1}\right)$ nas plantas em solo-controle e contaminado, respectivamente. $\quad *$ Significativamente diferentes $(\mathrm{P} \leq 5 \%)$. 
$\mathrm{Zn}$ nas espécies que cresceram em solo contaminado variou de 950 a $15.094 \mu$ planta $^{-1}$, indicando o comportamento muito diferenciado das espécies em relação ao acúmulo desse elemento. A maioria das espécies apresentou maior quantidade relativa de $\mathrm{Zn}$ nas raízes em solo contaminado, enquanto $M$. peruiferum $\mathrm{e}$ Piptadenia gonoacantha apresentaram maior quantidade relativa desse elemento nas folhas. Isso sugere que essas duas espécies apresentam alta capacidade de translocação do $\mathrm{Zn}$ absorvido. Espécies com essa característica podem facilitar o processo de distribuição do $\mathrm{Zn}$ nos componentes do ecossistema, tendo-se em vista que as folhas reciclam mais rapidamente que as demais partes da planta. Em C. lasiophylla, D. cuneatum, $M$. nictidans e $T$. micrantha no solo contaminado, verificou-se elevada quantidade relativa de $\mathrm{Zn}$ no caule. Esse acúmulo preferencial no caule pode contribuir para a imobilização do elemento na vegetação, tornando essas espécies promissoras para programas de fitorremediação, desde que essas tolerem as condições de contaminação.

Verificou-se efeito significativo $(\mathrm{P} \leq 0,05)$ da contaminação sobre o acúmulo total de $\mathrm{Cd}$ na maioria das espécies estudadas (Figura 3). O Cd acumulado nos tecidos foi muito baixo para todas as espécies crescidas no solo-controle, ao passo que no solo contaminado a quantidade total acumulada variou de 32 a $697 \mu$ g planta $^{-1}$, sendo as espécies D. cuneatum, $S$. granulosum-leprosum, $P$. dubium e $C$. fissilis as que apresentaram maior acúmulo total desse elemento. A elevada quantidade acumulada de $\mathrm{Cd}$ apresentada por $S$. granulosum-leprosum (557 $\mu \mathrm{g}$ planta $^{-1}$ ) deve-se principalmente à alta absorção desse elemento, que atingiu teores de $55 \mathrm{mg} \mathrm{kg}^{-1}$ na matéria seca das folhas (Tabela 2). D. cuneatum também apresentou elevado acúmulo total de $\mathrm{Cd}\left(697 \mu \mathrm{g}\right.$ planta $\left.^{-1}\right)$, e a maior parte desse elemento ficou acumulada no caule $(63 \%)$ em virtude principalmente do elevado teor desse elemento no caule (Tabela 2). Para as demais espécies, houve maior distribuição do $\mathrm{Cd}$ acumulado nas raízes por causa dos elevados teores observados.
$\mathrm{O}$ acúmulo total de $\mathrm{Cu}$ nas espécies foi pouco influenciado pela contaminação do solo (Figura 4) em comparação a $\mathrm{Zn}$ e $\mathrm{Cd}$. Algumas espécies como $C$. lasiophylla, D. cuneatum e $S$. macranthera apresentaram maior acúmulo de $\mathrm{Cu}$ no controle. Esse comportamento indica que o acúmulo de $\mathrm{Cu}$ sofreu interferência de outros íons no processo de absorção, pois verificou-se que os valores acumulados não foram crescentes com a contaminação, mesmo o $\mathrm{Cu}$ tendo apresentado alta concentração no solo contaminado (Quadro 1). Uma provável interferência na absorção de $\mathrm{Cu}$ foi a elevada contaminação do solo por $\mathrm{Zn}$, uma vez que altas atividades de $\mathrm{Zn}^{2+}$ na solução do solo promovem efeito antagônico na absorção do $\mathrm{Cu}^{2+}$ (Alloway, 1993). O acúmulo de $\mathrm{Pb}$ nos tecidos foi pouco influenciado pela contaminação do solo, verificando-se maior acúmulo relativo nas raízes (dados não apresentados). Segundo Kahle (1993), o elevado acúmulo de $\mathrm{Pb}$ nas raízes pode estar relacionado com a imobilização desse elemento por meio de polímeros orgânicos insolúveis presentes no tecido.

Verifica-se, portanto, que a contaminação do solo influenciou o crescimento e o teor de metais pesados na matéria seca, apresentando teores de $\mathrm{Zn}$ e $\mathrm{Cd}$ nas folhas mais elevados que a faixa considerada tóxica para o crescimento de diversas plantas, evidenciando o potencial fitotóxico desses elementos para as espécies arbóreas estudadas. Algumas espécies como $M$. nictidans, M. peruiferum, Piptadenia gonoacantha, $S$. macranthera e $T$. micrantha acumularam proporcionalmente mais $\mathrm{Zn}$ e $\mathrm{Cd}$ na parte aérea do que nas raízes (Figura 5), sendo essas espécies influenciadas negativamente pela contaminação. No entanto, a espécie $D$. cuneatum, que não foi influenciada pela contaminação, também apresentou elevado índice de translocação de Zn e Cd (53\% e $94 \%$, respectivamente), porém esses se acumularam mais no caule do que nas folhas (Figura 3). Isso pode indicar um mecanismo adicional de proteção da $D$. cuneatum, ocasionando menor impacto da contaminação em relação às 


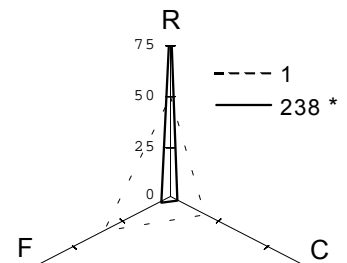

A. mangium

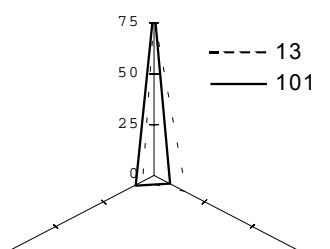

A. peregrina

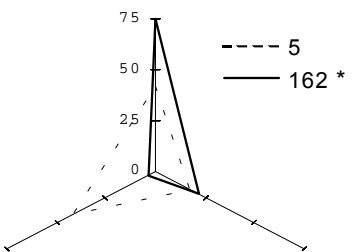

C. lasiophylla

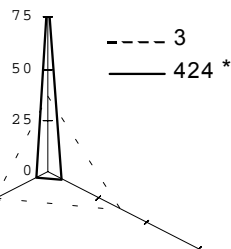

C. fis silis

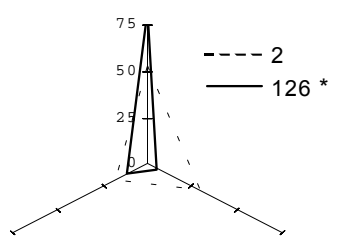

C. langsdorffi

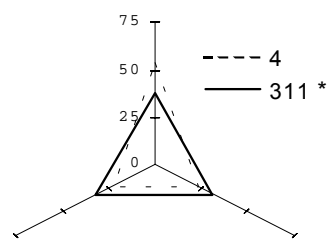

M. nictidans

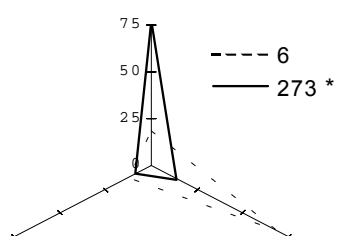

C. trichotoma

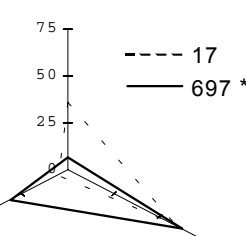

D. cuneatum

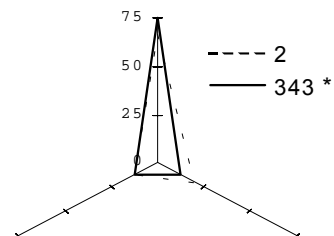

M. caesalpiniaefolia

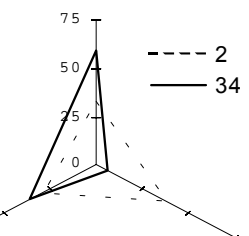

M. peruiferum

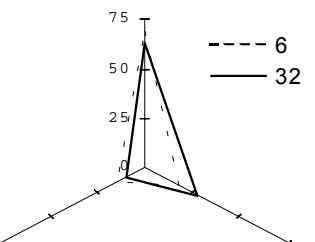

Plat. gonoacantha

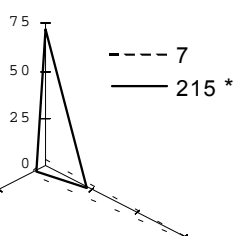

H. courbaril
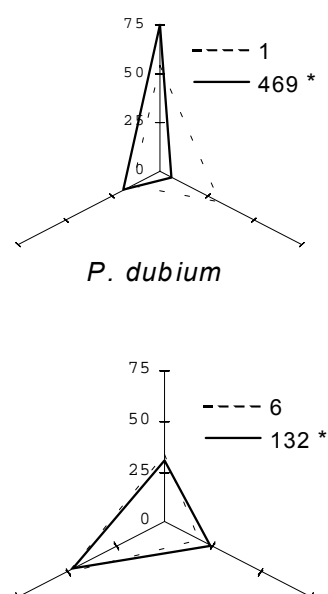

S. macranthera

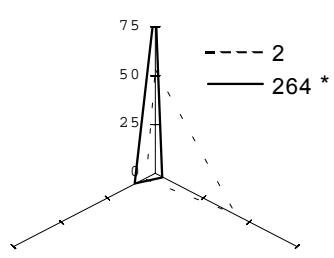

Pipt. gonoacantha

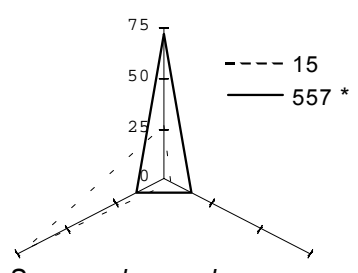

S. granulosum-leprosum

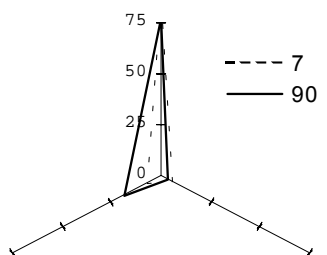

T. impetiginosa

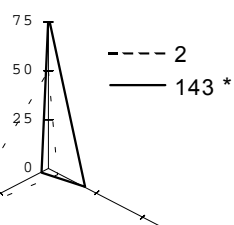

M. umbellata

FIGURA 3. Acúmulo e distribuição (\% do total acumulado) de Cd nas raízes (R), caule (C) e folhas (F) em mudas de espécies arbóreas. Linhas tracejadas e contínuas representam o total acumulado ( $\mu$ g planta $\left.{ }^{-1}\right)$ nas plantas em solo-controle e contaminado, respectivamente.

* Significativamente diferentes $(\mathrm{P} \leq$ $5 \%)$ 


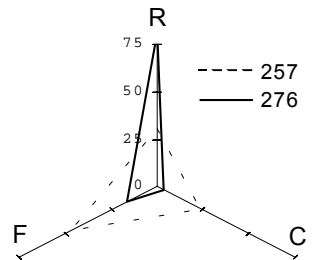

A. mangium

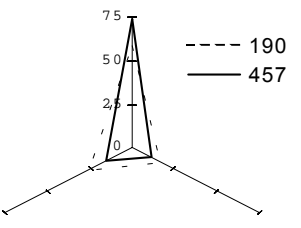

C. langsdorffi

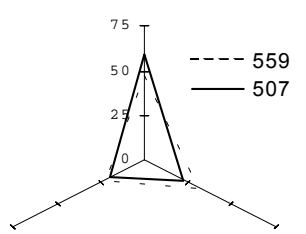

M. nictidans

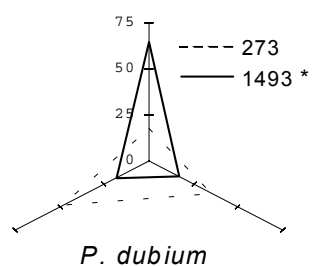

P. dubium

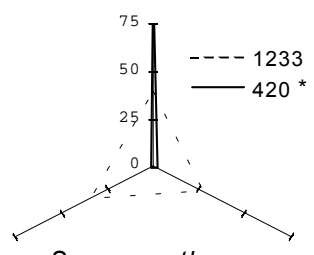

S. macranthera

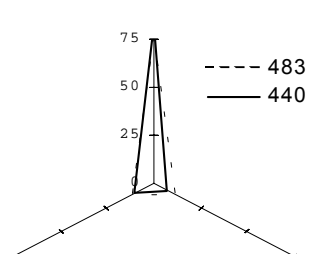

A. peregrina

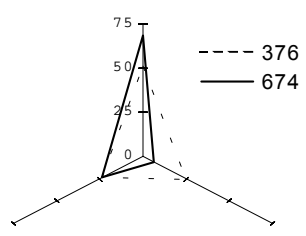

C. trichotoma
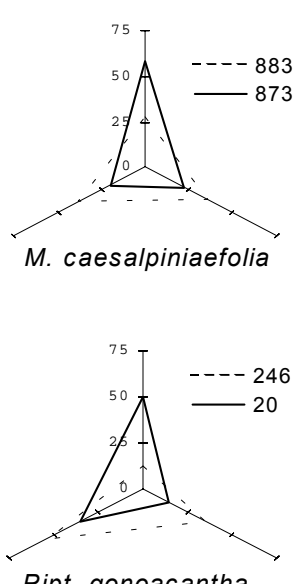

Pipt. gonoacantha

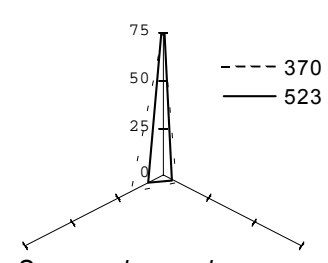

S. granulosum-leprosum

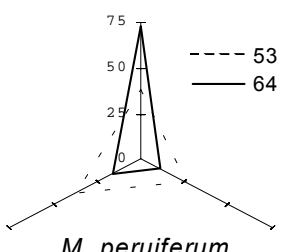

M. peruiferum

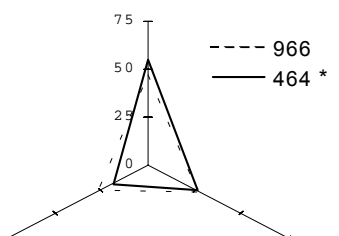

C. lasiophylla

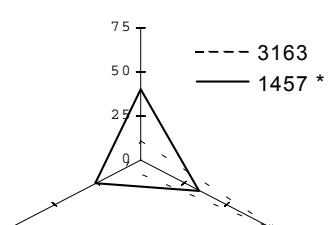

D. cuneatum

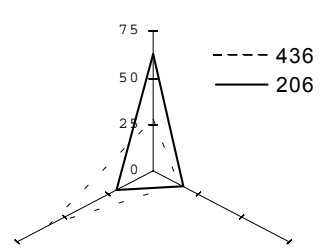

Plat. gonoacantha

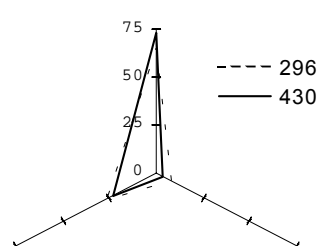

T. impetiginosa
H. courbaril

S. schottiana

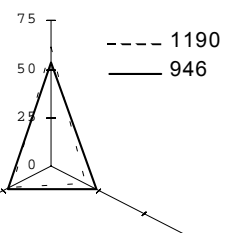

C. fissilis
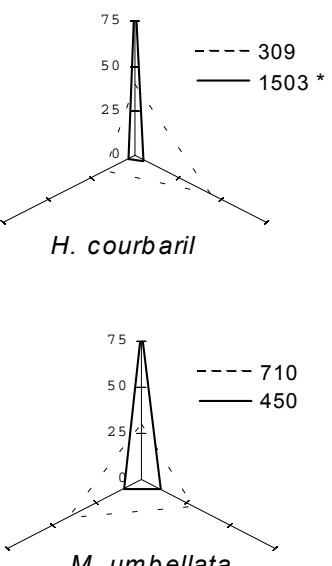

M. umbellata
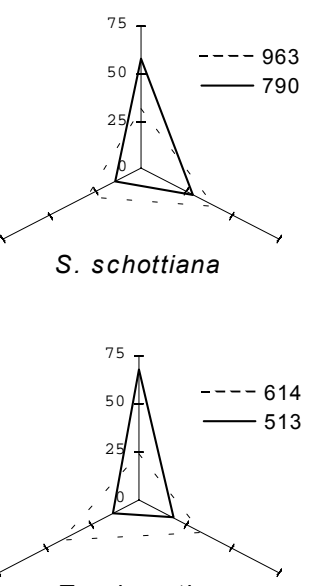

T. micrantha

FIGURA 4. Acúmulo e distribuição (\% do total acumulado) de $\mathrm{Cu}$ nas raízes $(\mathrm{R})$, caule $(\mathrm{C})$ e folhas (F) em mudas de espécies arbóreas. Linhas tracejadas e contínuas representam o total acumulado ( $\mu g$ planta $\left.{ }^{-1}\right)$ nas plantas em solo-controle e contaminado, respectivamente. * Significativamente diferentes $(\mathrm{P} \leq$ $5 \%)$. 


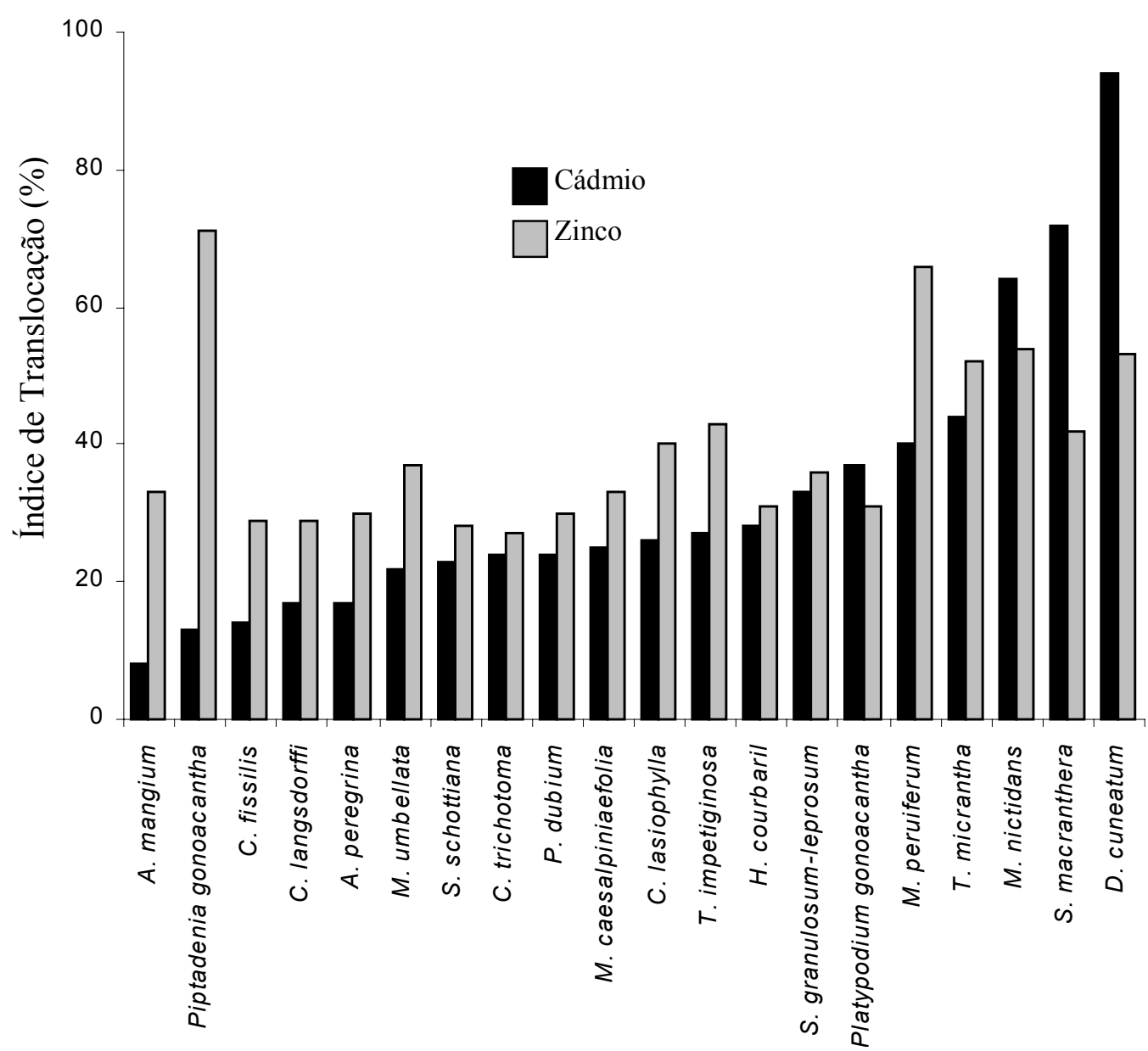

FIGURA 5. Índice de translocação de $\mathrm{Zn}$ e $\mathrm{Cd}$ em mudas de espécies arbóreas crescidas em solo contaminado com metais pesados.

espécies que apresentaram maior acúmulo nas folhas. Enquanto isso, algumas espécies como $C$. fissilis, C. langsdorffi e $A$. mangium apresentaram baixa translocação de $\mathrm{Zn}$ e $\mathrm{Cd}$, o que pode estar contribuindo para a baixa sensibilidade dessas espécies à contaminação do solo.

\section{CONCLUSÕES}

1. Ainda que esperados, porém não disponíveis na literatura, são apresentados resultados que demonstram que as mudas de espécies arbóreas estudadas comportam-se diferenciadamente em relação aos teores e acúmulo de metais pesados em suas diferentes partes.

2. Mesmo tendo-se comparado mudas com diferentes idades, o padrão de distribuição de $\mathrm{Zn}$ e $\mathrm{Cd}$ nas diferentes partes das plantas se relaciona com o comportamento dessas ao excesso de metais pesados no solo.

3. As espécies $M$. nictidans, $M$. peruiferum, Piptadenia gonoacantha, $S$. macranthera e $T$. micrantha foram as que mais translocaram $\mathrm{Zn}$ e/ou Cd para a parte aérea, sendo afetadas negativamente pela contaminação. 
4. Embora $D$. cuneatum também tenha apresentado elevada translocação de $\mathrm{Zn}$ e Cd para a parte aérea, houve maior acúmulo desses elementos no caule. Isso parece ter contribuído para um impacto reduzido da contaminação em comparação às espécies que apresentaram maior quantidade relativa desses elementos nas folhas.

5. A baixa sensibilidade de A. mangium, C. langsdorffi e C. fissilis à contaminação do solo relaciona-se com a baixa translocação de $\mathrm{Zn}$ e $\mathrm{Cd}$.

\section{LITERATURA CITADA}

ABICHEQUER, A.D.; BOHNEN, H. Eficiência de absorção, translocação e utilização de fósforo por variedades de trigo. Revista Brasileira de Ciência do Solo, 22:21-26, 1998.

ACCIOLY, A.M.A.; SIQUEIRA, J.O. Contaminação química e biorremediação do solo. Tópicos em Ciência do Solo, 1:299-351, 2000.

ALLOWAY, B.J. Heavy metals in soils. New York: John Wiley, 1993. 339p.

ARDUINI, I.; GODBOLD, D.L.; ONNIS, A. Cadmium and copper uptake and distribution in Mediterranean tree seedlings. Physiologia Plantarum, 97:111-117, 1996.

BAKER A.J.M. Accumulators and excluders strategies in the response of plants to heavy metals. Journal of Plant Nutrition, 3:643654, 1981.

BARCELÓ, J.; POSCHENRIEDER, Ch. Respuestas de las plantas a la contaminación por metales pesados. Suelo y Planta, 2:345361, 1992.

BOTELHO, S.A.; DAVIDE, A.C.; PRADO, N.S.; FONSECA, E.M.B. Implantação de mata ciliar. Belo Horizonte, CEMIG; Lavras, UFLA, 1995. $28 \mathrm{p}$.

ELTROP, L.; BROWN, G.; JOACHIM, O.; BRINKMANN, K. Lead tolerance of betula and salix in the mining area of Mechernich/Germany. Plant and Soil, 131:279-285, 1991.
EMBRAPA. Serviço Nacional de Levantamento e Conservação de Solos (Rio de Janeiro, RJ). Manual de métodos de análise de solo. Rio de Janeiro, 1979. Não paginado.

FOSTER, J.C. Total heavy metal contents by aqua regia digestion. In: ALEF, K.; NANNIPIERI, P. (Eds.). Methods in applied soil microbiology and biochemistry. San Diego: Academic, 1995. p.101-102.

GUSSARSSON, M.; ADALSTEINSSON, P.J.; ASP, H. Cadmium and copper interactions on the accumulation and distribution of $\mathrm{Cd}$ and $\mathrm{Cu}$ in birch (Betula pendula Roth) seedlings. Plant and Soil, 171:185-187, 1995.

HUANG, J.W.; CUNNINGHAM, S.D. Lead phytoextraction: species variation in lead uptake and translocation. New Phytologist, 134: 75-84, 1996.

KABATA-PENDIAS, A.; PENDIAS, H. Trace elements in soils and plants. Boca Raton: CRC Press, 1985. 315p.

KAHLE, H. Response of roots of trees to heavy metals. Environmental and Experimental Botany, 33:99-119, 1993.

MARQUES, T.C.L.L. de S. e M.; MOREIRA, F.M. de S.; SIQUEIRA, J.O. Crescimento e teor de metais de mudas de espécies arbóreas cultivadas em solo contaminado com metais pesados. Pesquisa Agropecuária Brasileira, 35:121-132, 2000.

RIBEIRO FILHO, M.R.; CURI, N.; SIQUEIRA, J.O.;P MOTTA, P.E.F. Metais pesados em solos de rejeitos de indústria de processamento de zinco. Revista Brasileira de Ciência do Solo, 23:453-464, 1999.

SHAW, A.J. Heavy metal tolerance in plants: Evolutionary aspects. New York, CRC Press, 1989. 355p.

VERKLEIJ, J.A.C.; PAREST, J.E. Cadmium tolerance and co-tolerance in Silene vulgaris (Moench.) Garcke [= S. cucubalus (L.) wilb.]. The New Phytologist, 111:637-645, 1989. 